\title{
Resección hepática extendida con vena cava inferior por colangiocarcinoma intrahepático. Presentación de 2 casos
}

${ }^{1}$ Facultad de Medicina, Clínica Alemana Santiago-Universidad del Desarrollo.

${ }^{2}$ Hospital Barros LucoUniversidad de Chile. Santiago, Chile.

Recibido el 31 de julio de 2018 y aceptado para publicación el 4 de diciembre de 2018.

Correspondencia a: Felipe A. Castillo H. felipe.castillo10@gmail.com

\author{
Guillermo Rencoret P. ${ }^{1}$, Marcelo Vivanco L. ${ }^{1}$, Felipe A. Castillo H. ${ }^{1,2}$, \\ Giancarlo Schippacasse F. ${ }^{1}$, Xabier De Aretxabala U. ${ }^{1}$, \\ Víctor Bianchi S. ${ }^{1}$, Juan Hepp K. ${ }^{1}$ y Horacio Ríos R. ${ }^{1}$
}

\section{Extended hepatic resection with inferior cava vein for intrahepatic cholangiocarcinoma. Presentation of two cases}

Introduction: Intrahepatic cholangiocarcinoma is the second most common primary liver tumor and surgical resection the only valid curative treatment. Case reports: We describe two patients harboring an intrahepatic cholangiocarcinoma with cava vein involvement who underwent resection at Clinica Alemana of Santiago. Both patients were females with ages of 39 and 47 years old. Both patients underwent left liver resection, associated to resection of segment I and of a portion of cava vein. Reconstruction of resected portion of the cava vein was performed by using a pericardium bovine patch and primary closure respectively. Postoperative period was uneventfully being discharged at 13 and 23 days respectively. Discussion: Although surgical resection is the only way to get curativeness, frequent involvement of large vascular structures make treatment unfeasible. A multidisciplinary approach associated with a meticulous technique performed by an experienced surgical team make possible to accomplish the above objective. Key words: "Hepatic resection"; "Cholangiocarcinoma"; "Inferior cava vein".

\section{Resumen}

Introducción: El colangiocarcinoma intrahepático $(\mathrm{CIH})$ corresponde al segundo tumor hepático primario y la resección quirúrgica es la única alternativa válida para el tratamiento curativo de esta enfermedad. Reporte de casos: Describimos 2 paciente portadores de CIH con compromiso de vena cava inferior (VCI) que fueron sometidos a resección en Clínica Alemana de Santiago (CAS). Ambas pacientes son de género femenino de 39 y 47 años de edad. Ambas fueron sometidas a resección mayor hepática izquierda, asociada a resección del segmento I y extendida a VCI. La reconstrucción de la VCI fue realizada con parche pericárdico bovino y cierre primario respectivamente. El período desde el posoperatorio hasta el alta, fue de 13 y 23 días respectivamente. Discusión: Aunque la reseccion quirúrgica es la única vía para la curación en el $\mathrm{CIH}$, el compromiso de estructuras vasculares hacen que esto no sea posible. El manejo multidisciplinario asociado a una técnica meticulosa realizada por un equipo quirúrgico experimentado, hacen posible lograr buenos resultados.

Palabras clave: resección hepática; colangiocarcinoma; vena cava inferior.

\section{Introducción}

El colangiocarcinoma es un tumor infrecuente, derivado de las células epiteliales de los conductos biliares. Sólo del 5 al $10 \%$ de éstos, son de ubicación intrahepática ${ }^{1}$. Los CIH corresponden al segundo cáncer primario del hígado (luego del hepa- tocarcinoma), y su incidencia a nivel mundial va en aumento $^{2}$. Se clasifican en proximales o periféricos según su origen ${ }^{1}$. Clínicamente pueden presentar historia de dolor inespecífico en el cuadrante superior derecho abdominal, pérdida de peso o alteración de pruebas hepáticas (principalmente fosfatasa alcalina), sin embargo, la mayoría son asintomáticos, lo 
que determina su diagnóstico en etapas avanzadas. Algunos de estos pacientes son diagnosticados incidentalmente por exámenes imagenológicos de rutina o en seguimiento de otras hepatopatías (infección por virus hepatitis $\mathrm{B}$ y C) ${ }^{3}$. La resección quirúrgica es la única opción de tratamiento curativo. En casos de pacientes con enfermedad localmente avanzada, puede existir compromiso de la vena cava inferior (VCI), siendo necesaria la resección y reconstrucción vascular concomitante a la resección hepática correspondiente ${ }^{4}$.

El objetivo de este trabajo, es describir dos casos de pacientes sometidos a RH extendida con resección y reconstrucción de la VCI, por CIH avanzado.

\section{Caso 1}

Mujer de 47 años, con antecedentes de hipotiroidismo, resistencia a la insulina y dislipidemia en tratamiento, consulta a CAS por 2 meses de dolor epigástrico con baja de peso no cuantificada. En estudio se realiza ecotomografía abdominal que evidencia lesión focal hepática de 6,5 centímetros, se complementa estudio con resonancia magnética (RM) de abdomen que evidencia masa hepática que compromete los segmentos I, IV y VIII, una segunda lesión satélite adyacente en segmento IV, con compresión de VCI retrohepática, sin características radiológicas específicas (Figura 1). Marcadores tumorales (alfafetoproteina (AFP), antígeno CA 19-9 (CA 19-9) y antígeno carcinoembrionario (CEA)) dentro de rangos normales. Se realiza biopsia hepática por punción evidenciando adenocarcinoma moderadamente diferenciado sugerente de colangiocarcinoma G2 (CK 7 +, CA 19-9 +). Se realiza quimioterapia intraarterial (QIA) con 5-fluoracilo de acción rápida a través de reservorio intrahepático, con 3 ciclos, lográndose reducción de tamaño tumoral. Se discute en comité oncológico (CO) donde se decide resolución quirúrgica.

El procedimiento quirúrgico se realiza a través de una laparotomía subcostal bilateral, se evidencia compromiso local de VCI retrohepática por tumor, se libera el hígado restante aislando la lesión, se realiza maniobra "Hanging" y se procede a hepatectomía izquierda + resección del segmento I. Se realiza control vascular parcial con pinza Satinsky longitudinal, bien tolerado por la enferma y se secciona pared de VCI comprometida. Se procede a reparación con parche de pericardio bovino sin incidentes (Figura 2).

Evoluciona bien en el postoperatorio, con manejo en Unidad de Cuidado Intensivos (UCI) por 3 días, y dándose de alta a los 23 días. Presentó fístula biliar evidenciada por drenaje, de alto débito, por lo que se realiza colangiopancreatografía endoscópica retrógrada (con instalación de prótesis y papilotomía) con resolución completa. Biopsia de pieza operatoria concluye colangiocarcinoma intrahepático, de 4,6 x $5 \times 3,7 \mathrm{~cm}$, ningún linfonodo con metástasis, bordes quirúrgicos libres y patrón de crecimiento pseudoconductillar.

Paciente con 15 meses de seguimiento, sin evidencia de recidiva ni progresión de enfermedad.

\section{Caso 2}

Mujer de 39 años, sin antecedentes mórbidos relevantes, consulta en otro centro por hallazgo incidental de tumor hepático en ecotomografía

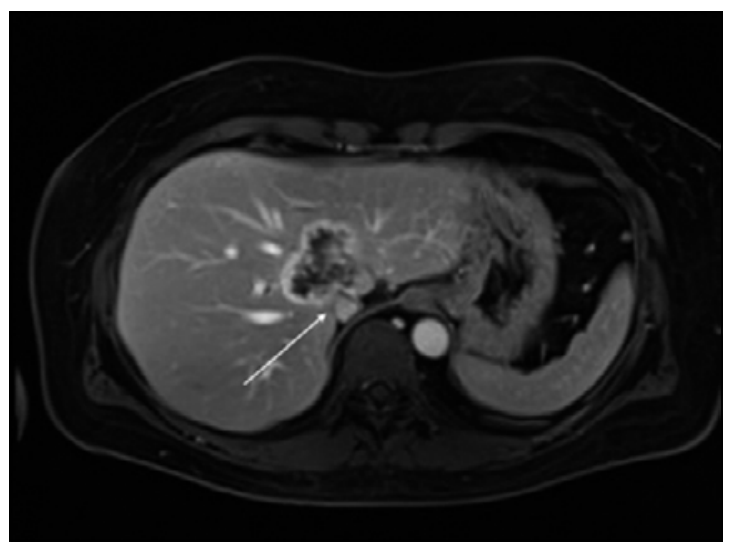

Figura 1. Imagen radiológica de tumor de caso 1 , la flecha muestra la compresión de vena cava inferior por tumor.

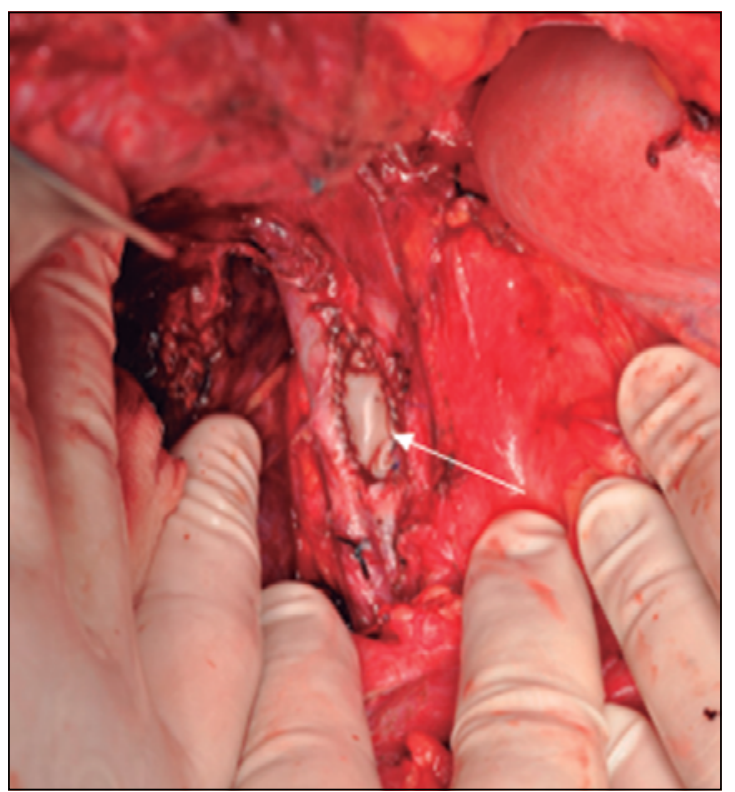

Figura 2. Imagen intraoperatoria de caso clínico 1, la flecha muestra parche de pericardio bovino. 


\section{CASOS CLÍNICOS}

abdominal que compromete el lóbulo hepático izquierdo. Se realiza biopsia incisional que confirma CIH. Se recibe en CAS donde se completa estudio con RM de hígado que evidencia tumor hepático que

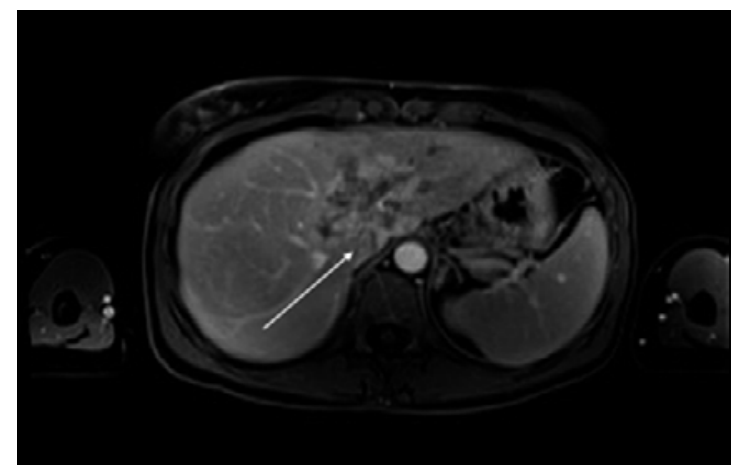

Figura 3. Imagen radiológica de tumor de caso 2, la flecha muestra la compresión de vena cava inferior por tumor.

Figura 4. Volumetría hepática post embolización portal izquierda.

Figura 5. Imagen intraoperatoria de caso 2 compromete segmentos I, II, III y IV (Figura 3). Se discute en $\mathrm{CO}$ y se decide neoadyuvancia con gemcitabina/cisplatino, en el control del tercer ciclo se determina mala respuesta, por lo que se rediscute y se decide resección quirúrgica previa embolización (por remanente hepático insuficiente). Se realiza embolización portal izquierda y selectivamente segmentos 5 y 6 , con respuesta satisfactoria (volumen remanente hepático 39\%) (Figura 4).

El procedimiento quirúrgico se realiza a través de una laparotomía subcostal bilateral, se evidencia gran tumor hepático con compromiso local VCI retrohepática, se procede a hepatectomía izquierda extendida (segmentos anteriores) + resección del segmento I. Se realiza control vascular parcial de VCI con pinza Satinsky longitudinal, bien tolerado por la enferma y se reseca tumor incluyendo la vena comprometida. Se procede a reparación con rafia primaria sin incidentes (Figura 5).

Evoluciona bien en el postoperatorio, con manejo en Unidad de Cuidado Intensivos (UCI) por 3 días, y dándose de alta a los 13 días, sin complicaciones. Biopsia pieza operatoria concluye colangiocarcinoma intrahepático, de 5,8 x 4,6 x 3,2 cm, ningún linfonodo con metástasis, bordes quirúrgicos venoso positivo de $3 \mathrm{~mm}$ y patrón de crecimiento pseudoconductillar. Posteriormente, recibió 3 ciclos de quimioterapia adyuvante con FOLFOX.

Paciente con 10 meses de seguimiento, sin evidencia de recidiva ni progresión de enfermedad.

\section{Discusión}

La cirugía tiene un rol fundamental en el tratamiento del $\mathrm{CIH}$, incluyendo los tumores localmente avanzados. La mayoría de los pacientes que se asocian a compromiso de VCI, mueren al año sino reciben tratamiento ${ }^{5}$.

El desarrollo de la cirugía hepática, tanto en trasplante como en nuevas técnicas de control vascular, ha permitido realizar resecciones extendidas en tumores avanzados, incluyendo aquellos que infiltran la VCI, otorgando una chance de cura a estos enfermos $^{6}$. En la literatura, centros de referencia han desarrollado esta experiencia no solo para $\mathrm{CIH}$, sino que también para otros tumores primarios y secundarios del hígado, como Arii et al. ${ }^{7}$, que describe 11 RE (10 mayores y 1 menores) o Nardo et al. ${ }^{8}$, con 19 RH (15 mayores y 4 menores) con sobrevida mediana de 71,2 y 54,3 meses respectivamente.

Las estrategias de control vascular son variadas (exclusión vascular hepática total o parcial), así como también el material utilizado para el reem- 
plazo vascular si es requerido (autólogo, xenólogo, protésico $)^{9}$; lo que ha determinado mejores resultados en la morbimortalidad de estos enfermos ${ }^{10}$. En nuestros casos ambos corresponden a resecciones parciales tangenciales de VCI; en el caso 1 se requirió pericardio bovino y en el caso 2 cierre primario con venorrafia.

En el caso 1, la paciente fue considerada irresecable en una primera instancia y se decidió neoadyuvancia con QIA. Algunas investigaciones han reportado la eficacia de la quimioterapia en este tipo de tumores ${ }^{11}$. En este caso la respuesta fue satisfactoria del punto de vista de tamaño tumoral e invasión local, lo que determinó la resecabilidad completa (R0) en el tiempo quirúrgico. Sin embargo, en el caso 2 , la respuesta a la quimioterapia sistémica fue nula, por lo que se decidió la resección quirúrgica. Debemos recalcar que la quimioterapia, por sí sola, no corresponde a una opción curativa ${ }^{12}$.

En conclusión, consideramos que la resolución quirúrgica de pacientes con $\mathrm{CIH}$ con compromiso de VCI es una alternativa válida y segura de ejecutar, en centros con experiencia en cirugía hepática. Las terapias adyuvantes podrían tener un rol relevante en el tratamiento de esta enfermedad.

\section{Responsabilidades éticas}

Protección de personas y animales. Los autores declaran que para esta investigación no se han realizado experimentos en seres humanos ni en animales.

Confidencialidad de los datos. Los autores declaran que en este artículo no aparecen datos de pacientes.

Conflictos de interés: no hay.

\section{Bibliografía}

1. Nagorney DM, Pawlik TM, Chun YS. Perihilar bile ducts. In: AJCC Cancer Staging Manual, 8th, Amin MB. (Ed), AJCC, Chicago 2017. P.311.

2. Saure A. Resultados del tratamiento quirúrgico del colangiocarcinoma intrahepático. Rev Chil Cir. 2016;68:15463.

3. Brown KM, Parmar AD, Geller DA. Intrahepatic Cholangiocarcinoma.Surg Oncol Clin N Am. 2014;23:231-46. doi:10.1016/j.soc.2013.10.004.

4. Malde DJ, Khan A, Prasad KR, Toogood GJ, Lodge JP. Inferior vena cava resection with hepatectomy: challenging but justified. HPB (Oxford) 2011;13:802-10.

5. Rougier P, Milan C, Lazorthes F, Fourtanier G, Partensky C, Baumel H, et al. Prospective study of prognostic factors in patients with unresected hepatic metastases from colorectal cancer. Fondation Francaise de Cancerologie Digestive. Br J Surg. 1995;82:1397-400.

6. Delis SG, Madariaga J, Ciancio G. Combined liver and inferior vena cava resection for hepatic malignancy. J Surg Oncol. 2007;96:258-64.

7. Arii S, Teramoto K, Kawamura T, Takamatsu S, Sato E, Nakamura N, et al. Significance of hepatic resection combined with inferior vena cava resection and its reconstruction with expanded polytetrafluorethylene for treatment of liver tumors. J Am Coll Surg. 2003;196:243-9.

8. Nardo B, Ercolani G, Montalti R, Bertelli R, Gardini A, Beltempo P, et al. Hepatic Resection for Primary or Secondary Malignancies with Involvement of the Inferior Vena Cava: Is This Operation Safe or Hazardous? J Am Coll Surg. 2005;201:671-9.
9. Malde DJ, Khan A, Prasad KR, Toogood GJ, Lodge JP. Inferior vena cava resection with hepatectomy: challenging but justified. HPB 2011;13:802-10.

10. Sano T, Shimada K, Nara S, Esaki M, Sakamoto Y, Kosuge T. Hepatobiliary resection with inferior vena cava resection and reconstruction using an autologous patch graft for intrahepatic cholangiocarcinoma. Langenbecks Arch Surg. 2008;393:599-603.

11. Shen WF, Zhong W, Liu Q, Sui CJ, Huang YQ, Yang JM. Adjuvant transcatheter arterial chemoembolization for intrahepatic cholangiocarcinoma after curative surgery: retrospective control study. World J Surg. 2011;35:2083-91.

12. Valle J, Wasan H, Palmer DH, Cunningham D, Anthoney A, Maraveyas $A$, et al. Cisplatin plus gemcitabine versus gemcitabine for biliary tract cancer. N Engl J Med. 2010;362:1273-81. 